\title{
14-bp Insertion/Deletion Polymorphism of the HLA-G gene in Breast Cancer among Women from North Western Iran
}

\author{
Mehdi Haghi ${ }^{1}$, Mohammad Ali Hosseinpour Feizi ${ }^{1 *}$, Majid Sadeghizadeh ${ }^{2}$, Abbas \\ Sahebghadam Lotfi ${ }^{3}$
}

\begin{abstract}
Background: The human leukocyte antigen-G (HLA-G) gene is highly expressed in cancer pathologies and is one strategy used by tumor cells to escape immune surveillance. A 14-bp insertion/deletion (InDel) polymorphism of the HLA-G gene has been suggested to be associated with HLA-G mRNA stability and the expression of HLA-G. The aim of present study was to assess any genetic association between this polymorphism and breast cancer among Iranian-Azeri women. Materials and Methods: In this study 227 women affected with breast cancer, in addition to 255 age-sex and ethnically matched healthy individuals as the control group, participated. Genotyping was performed using polymerase chain reaction and electrophoresis assays. The data were compiled according to the genotype and allele frequencies, compared using the Chi-square test. Statistical significance was set at $\mathbf{P}<0.05$. Results: In this case-control study, no significant difference was found between the case and control groups at allelic and genotype levels, although there is a slightly higher allele frequency of HLA-G 14bp deletion in breast cancer affected group. However,when the stage I subgroup was compared with stage II plus stage III subgroup of affected breast cancer, a significant difference was seen with the 14 bp deletion allele frequency. The stage II-III subgroup patients had higher frequency of deletion allele $(57.4 \%$ vs $45.8 \%)$ than stage I cases $(\chi 2=4.16$, $p$-value $=0.041)$. Conclusions: Our data support a possible action of HLA-G 14bp InDel polymorphism as a potential genetic risk factor for progression of breast cancer. This finding highlights the necessity of future studies of this gene to establish the exact role of HLA-G in progression steps of breast cancer.
\end{abstract}

Keywords: Breast cancer - polymorphism- HLA-G

Asian Pac J Cancer Prev, 16 (14), 6155-6158

\section{Introduction}

Breast cancer is the most commonly diagnosed cancer in women worldwide. In Iran, it ranks first among cancers diagnosed in women and is the fifth most common cause of death. It is complex and the causes of breast cancer are not fully known, likely caused by a combination of risk factors (Taghavi et al., 2012).

The immune system identifies cancerous and/or precancerous cells on the basis of tumor-specific antigens or molecules induced by cellular stress and eliminate them before they can cause harm. This process is referred to tumor immune surveillance theory (Burnet, 1957). But various changes take place in the microenvironment around the developing tumor, which some of these changes originated by tumor cells may help to escape the immune surveillance and favorable for tumor development and progression of cancer (Elliott et al., 2011). One such discovered molecule is HLA-G, which has been reported to have immunosuppressive and immunomodulatory roles in the cancer development (Tripathi et al., 2006).
The HLA-G non classical MHC class I protein exhibits a more restricted tissue distribution; it has been originally described as being selectively expressed in placental trophoblast (Ellis et al., 1986; Kovats et al., 1990). It found that HLA-G can be important in preventing maternal immune response against the fetus and placenta unit having different genetics as the immune tolerance in pregnancy mechanism (Larsen and Hviid, 2009)

HLA-G exhibits the immune tolerant function by inhibiting different immune-competent cells. This inhibitory effect is mediated by direct binding to inhibitory receptors. Thus, HLA-G can directly interact with different immune cell subpopulations including NK cells, $\mathrm{T}$ cells and professional antigen-presenting cells (APC), as well as endothelial cells via these receptors. This interaction is accompanied by the generation and maintenance of tolerance at different stages of the immune response, e.g., differentiation, proliferation, cytolysis and cytokine secretion. Next to these direct effects, HLA-G can exert its immune suppressive activity by indirect mechanisms. The knowledge gained from research into

${ }^{1}$ Department of Genetics, Faculty of Natural Sciences, University of Tabriz, ${ }^{2}$ Department of Genetics, Faculty of Biological Sciences, ${ }^{3}$ Department of Clinical Biochemistry, Faculty of Medical Sciences, Tarbiat Modarres University, Tehran, Tabriz, Iran *For correspondence:pourfeizi@eastp.ir,mehdihaghi@tabrizu.ac.ir 
the mechanisms of maternal immune tolerance lead to a greater understanding of the pathological processes including immunosuppressive mechanism in the cancer development (Amiot et al., 2011; Curigliano et al., 2013).

Several studies have shown that certain polymorphisms in HLA-G gene are associated with pregnancy complications such as preeclampsia and recurrent miscarriage (Wang et al., 2013; Enghelabifar et al., 2014) and cancer patient (Moon et al., 2011; Eskandari et al., 2013). The 14bp InDel polymorphism located in exon 8 of the 3' untranslated region (3' UTR) of the HLA-G gene was firstly reported in 1993 (Harrison et al., 1993). The aim of this case-control study was to determine association between the 14bp InDel polymorphism with breast cancer in Azeri population of northwestern-Iranian women.

\section{Materials and Methods}

After informed consent, EDTA-whole blood was collected from 227 women affected to breast cancer and 255 age-sex and ethnically matched healthy individuals as a control group. All the samples both patients and control group were from north western Iran population. Genomic DNA was extracted from peripheral white blood cells using Proteinas $\mathrm{K}$, then, genotyping of HLA-G was performed by polymerase chain reaction (PCR) with primers flanking the 14-nucleotide InDel polymorphism in the HLA-G gene. The sequences of primers used for amplification were: Forward primer: 5'GTGATGGGCTGTTTAAAGTGTCACC -3' and Reverse primer: 5'- GGAAGGAATGCAGTTCAGCATGA -3'.

PCR reaction in a final volume of $25 \mu 1$ contain 0.2 $\mathrm{mM}$ of each dNTP, $2.5 \mathrm{mM} \mathrm{MgCl} \neg 2,2.5 \mu \mathrm{l}$ of 10X PCR Buffer (pH 8.5) , $0.1 \mu \mathrm{M}$ of both primers, 1 Unit Taq polymerase, and finally $2 \mu 1$ of template DNA was added per reaction. PCR cycling conditions were as follows: an initial denaturation at $94^{\circ} \mathrm{C}$ for $3 \mathrm{~min}$, followed by a three step cycle of $20 \mathrm{sec}$ at $94^{\circ} \mathrm{C}, 20 \mathrm{sec}$ at $58^{\circ} \mathrm{C}$, and $30 \mathrm{sec}$ at $72^{\circ} \mathrm{C}$ for 30 cycles, and ending with a final extension of $3 \mathrm{~min}$ at $72^{\circ} \mathrm{C}$ in a PCR System thermal cycler. The PCR reaction for the insertion type allele product the 224bp amplicon, and the 210bp for allele contained 14 bp deletion polymorphism. The PCR products depending on the absence or presence of the $14 \mathrm{bp}$ were analyzed by electrophoresis in $15 \%$ gel of polyacrylamide and stained with ethidium bromide. (Figure 1). In this study, genotype and allele frequencies were compared using $\chi 2$ test. It was set statistical significance at $\mathrm{P}<0.05$ for tests.

\section{Results}

The frequencies of the allelic variant and genotype of studied polymorphism in case and control groups are summarized in Table 1. HLA-G 14bp deletion allel frequency in breast cancer patients was $52.6 \%$ and $50.6 \%$ among controls, and the difference was not statistically significant $\left(\chi^{2}=0.41, p\right.$-value $\left.=0.524\right)$. Also no significant difference was found at genotype level $\left(\chi^{2}=0.49, p\right.$-value $\left.=0.485\right)$. In case group, the genotype distribution showed $24.7 \%, 55.9 \%$, and $19.4 \%$ for Del/ Del, Del/Ins, and Ins/Ins genotypes, respectively. It
Table 1. Genotype Distribution and Allele Frequencies of HLA-G 14 bp InDel Gene Polymorphisms in Breast Cancer Patients and Control Individuals

\begin{tabular}{|c|c|c|c|c|}
\hline & Control & Case & $\chi^{2}$ & $\mathrm{p}$ value \\
\hline \multicolumn{5}{|c|}{ Genotypes } \\
\hline$\Delta \Delta$ & $52(20.4 \%)$ & $56(24.7 \%)$ & 0.49 & 0.485 \\
\hline$\Delta+$ & $154(60.4 \%)$ & $127(55.9 \%)$ & & \\
\hline++ & $49(19.2 \%)$ & $44(19.4 \%)$ & & \\
\hline \multicolumn{5}{|c|}{ Alleles } \\
\hline$\Delta$ & $258(50.6 \%)$ & $239(52.6 \%)$ & 0.41 & 0.524 \\
\hline+ & $252(49.40 \%)$ & $215(47.4 \%)$ & & \\
\hline
\end{tabular}

Table 2. Genotype Distribution and Allele Frequencies of HLA-G 14 bp InDel Gene Polymorphisms in Stage I and Stage II-III Subgroup of Breast Cancer Patients

\begin{tabular}{|c|c|c|c|c|}
\hline & Stage I & Stage II-III & $\chi^{2}$ & $\mathrm{p}$ value \\
\hline \multicolumn{5}{|c|}{ Genotypes } \\
\hline$\Delta \Delta$ & $11(18.7 \%)$ & $32(29.6 \%)$ & 4.65 & 0.031 \\
\hline$\Delta+$ & $32(54.2 \%)$ & $60(55.6 \%)$ & & \\
\hline++ & $16(27.1 \%)$ & $16(14.8 \%)$ & & \\
\hline \multicolumn{5}{|c|}{ Alleles } \\
\hline$\Delta$ & $54(45.8 \%)$ & $124(57.4 \%)$ & 4.16 & 0.041 \\
\hline
\end{tabular}

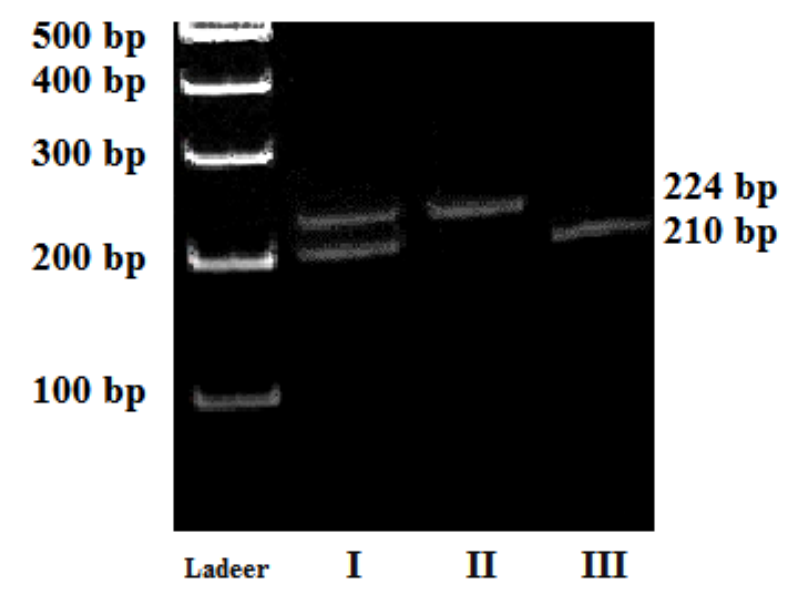

Figure 1. Polyacrylamide Gel Electrophoresis of PCR Production Containing HLA-G 14 bp InDel Polymorphism. I: Del/Ins genotype; II: Del/Del genotype; III: Ins/Ins genotype

was $20.4 \%, 60.4 \%$ and $19.2 \%$ for control group. But remarkable result was when compared the stage I with stage II-III subgroup of breast cancer patients (Table 2). The stage II and III subgroup patient had higher frequency of deletion allele $(57.4 \%$ vs $45.8 \%)$ than stage I patient $\left(\chi^{2}=4.16, \mathrm{p}\right.$-value $\left.=0.041\right)$. Also the frequency of Del/ Del, Del/Ins and Ins/Ins genotypes were $18.7 \%, 54.2 \%$ and $27.1 \%$ in stage I patient subgroup, whereas it was $29.6 \%, 55.6 \%$ and $14.8 \%$ in stage II-III patient subgroup $\left(\chi^{2}=4.65, p\right.$-value $\left.=0.031\right)$. Therefore, our result revealed significant differences at both allele and genotype of this polymorphism between stage I and II-III of breast cancer patients.

\section{Discussion}

The prevalence of breast cancer has been increased in Iran in recent years. In addition, the age of breast cancer 
among Iranian women has been decreased. Even though it is as a result of development methods of screening, the molecular study is necessary in order to improve breast cancer control and treatment. (Somi et al., 2009; Eivazi et al., 2011; Aflakseir and Abbasi, 2012; Shahidi et al., 2014)

The focus of some recently publications is polymorphism in the HLA-G gene and protein and its possible importance in expression, function, and disease associations (Larsen and Hviid, 2009). HLA-G appears to play a role in the suppression of immune responses and contribute to long-term immune escape or tolerance. HLA-G expression can be induced in different diseases including cancers (Elliott et al., 2011; Tuncel et al., 2013).

Compared with the classical HLA class-I molecules, limited HLA-G coding region polymorphisms have been reported in the human population, whereas a relatively higher degree of variation is known in its promoter and $3^{\prime}$ untranslated region (3'-UTR) (Castelli et al., 2014; Kuroshli et al., 2014). The HLA-G 3'-UTR contains regulatory elements included polyadenylation signals and AU-rich sequences (Castelli et al., 2010), which play important role in the spatial and temporal expression of HLA-G (Gonzalez et al., 2012). Therefore, the different polymorphisms located in the HLA-G $3^{\prime}$-UTR can influence the HLA-G expression.

The 14-bp InDel polymorphism located in exon 8 of the $3^{\prime}$ UTR is present in both the HLA-G gene and the transcript. It has been documented that $14 \mathrm{bp}$ InDel polymorphism not only has been associated with the expression of HLA-G but also effect to RNA splicing pattern and its stability (Rousseau et al., 2003). There is growing documents to support the viewpoint that some miRNA specifically regulate HLA-G expression by targeting its 3'UTR (Veit and Chies, 2009; Manaster et al., 2012). The $14 \mathrm{bp}$ deletion allele has been associated with high expression of HLA-G mRNA, whereas The $14 \mathrm{bp}$ Ins allele (5'-ATTTGTTCATGCCT-3') has been associated with low expression of HLA-G and low production of most mRNA isoforms (Hviid et al., 2004; Martelli et al., 2013; Jeong et al., 2014)

The 14bp InDel polymorphism has been extensively studied in many different publications (Moon et al., 2011; Eskandari et al., 2013). In research on south-east Iranian population, suggest that the 14-bp InDel polymorphism in HLA-G gene could be a genetic risk factor for the susceptibility to breast carcinoma (Eskandari et al., 2013). The meta-analysis study suggested that HLA-G 14-bp InDel polymorphism might contribute to breast cancer susceptibility and overall cancer risk among Asians (Yz et al., 2014). In Azeri population of Iran located north western Iran, There is growing study performed that tried to access the association between some polymorphisms and breast cancer (Pouladi et al., 2014; Saadatian et al., 2014; Sedaie et al., 2014). Until, in this population there is no report on HLA-G polymorphisms in breast cancer.

In this case-control, no significant difference was found at allelic and genotype levels between the case and control groups, although there is a slightly higher allele frequency of HLA-G 14bp deletion in breast cancer affected group. But when the stage I subgroup compared with stage II plus stage III subgroup of affected breast cancer, It can be seen the significant difference with 14 bp deletion allele frequency. This finding extracted from comparison allele and genotypes frequencies between stages is the first report. It seems the $14 \mathrm{bp}$ deletion allele or linked haplotype, because of high expression of HLA-G, can go forward the tumor cells to progressive stage of breast cancer. In conclusion, our data support a possible action of HLA-G 14bp InDel polymorphism as a potential genetic risk factor for progression of breast cancer. This finding can highlight this gene as candidate to future more studies to establish the exact role of HLA-G in progression steps of breast cancer.

\section{References}

Aflakseir A, Abbasi P (2012). Health beliefs as predictors of breast cancer screening behavior in a group of female employees in Shiraz. Iranian J Cancer Prev, 5, 124-9.

Amiot L, Ferrone S, Grosse-Wilde H, Seliger B (2011). Biology of HLA-G in cancer: a candidate molecule for therapeutic intervention? Cell Mol Life Sci, 68, 417-31.

Burnet FM (1957). Cancer- a biological approach: I. the processes of control. II. the significance of somatic mutation. Brit Med Jour, 1, 779-786.

Castelli EC, Mendes-Junior CT, Deghaide NH, et al (2010). The genetic structure of 3'untranslated region of the HLA-G gene: polymorphisms and haplotypes. Genes Immun, 112, 134-41.

Castelli EC, Ramalho J, Porto I, Lima T, et al (2014). Insights into HLA-G genetics provided by worldwide haplotype diversity. Frontiers in Immunology, 5, 476.

Curigliano G, Criscitiello C, Lucia Gelao L, Goldhirsch A (2013). Molecular pathways: Human Leukocyte Antigen G (HLA-G). Clin Cancer Res, 19, 5564-71

Eivazi-Ziaei J, Dastgiri S, Asvadi Kermani I, et al (2011). Age pattern of the occurrence of breast cancer in the northwest of Iran. Indian J Cancer, 48, 406-9

Elliott RL, Jiang XP, Phillips JT, Barnett BG, Head JF (2011). Human leukocyte antigen $\mathrm{G}$ expression in breast cancer: role in immunosuppression. Cancer Biother Radiopharm, 26, 153-7

Ellis SA, Sargent IL, Redman CW, McMichael AJ (1986). Evidence for a novel HLA antigen found on human extravillous trophoblast and a choriocarcinoma cell line. Immunology, 59, 595-601.

Enghelabifar M, Allafan S, Khayatzadeh J (2014). Association of the maternal 14-bp insertion/deletion polymorphism in the histocompatibility leukocyte antigen $\mathrm{G}$ gene with recurrent implantation failure. Iran J Reprod Med, 12, 641-6.

Eskandari-Nasab E, Hashemi M, Hasani SS, et al (2013). Association between HLA-G 3'UTR 14-bp ins/del polymorphism and susceptibility to breast cancer. Cancer Biomarkers, 13, 253-9.

Gonzalez A, Rebmann V, LeMaoult J, et al (2012). The immunosuppressive molecule HLA-G and its clinical implications. Crit Rev Clin Lab Sci, 49, 63-84.

Harrison GA, Humphrey KE, Jakobsen IB, Cooper DW (1993). A 14 bp deletion polymorphism in the HLA-G gene. Hum Mol Genet, 2, 2200.

Hviid TV, Larsen LG, Hoegh AM, Bzorek M (2004). HLA-G expression in placenta in relation to HLA-G genotype and polymorphisms. Am J Reprod Immunol, 52, 212-7.

Jeong S, Seho Park S, Park B, et al (2014). Human Leukocyte Antigen-G (HLA-G) polymorphism and expression in breast cancer patients. PlosOne, 9, 98284

Kovats S, Main EK, Librach C, et al (1990). Class I antigen, 
HLA-G, expressed in human trophoblasts. Science, $\mathbf{2 4 8}$, 220-3.

Kuroshli Z, Gourabi H, Bazrgar M, et al (2014). HLA-G allele and haplotype frequencies in a healthy population of Iran. Iran J Allergy Asthma Immunol, 13, 207-13.

Larsen MH, Hviid TV (2009). Human leukocyte antigen-G polymorphism in relation to expression, function, and disease. Hum Immunol, 70, 1026-34.

Manaster I, Goldman-Wohl D, Greenfield C (2012). MiRNAmediated control of HLA-G expression and function. PlosOne ,7, 33395.

Martelli G, Pancotto JA, Muniz YC, et al (2013). Polymorphic sites at the 3' untranslated region of the HLA-G gene are associated with differential hla-g soluble levels in the Brazilian and French population. PlosOne, 8, 71742

Moon A, Kim S, Ghung J, et al (2011). 14-bp Insertion/Deletion polymorphism of the HLA-G Gene in Osteosarcoma Patients. The Korean Journal of Pathology, 45, 485-490.

Pouladi N, Kouhsari SM, Feizi MH, et al (2014). Lack of association of intron 316 bp polymorphism of TP53 with breast cancer among Iranian-Azeri patients. Asian Pac J Cancer Prev, 15, 2631-4.

Rousseau P, Le Discorde M, Mouillot G, et al (2003).The 14 bp deletion-insertion polymorphism in the 3' UT region of the HLA-G gene influences HLA-G mRNA stability. Hum Immunol, 64, 1005-10.

Saadatian Z, Gharesouran J, Ghojazadeh M, et al (2014). Association of rs1219648 in FGFR2 and rs1042522 in TP53 with premenopausal breast cancer in an Iranian Azeri population. Asian Pac J Cancer Prev, 15, 7955-8.

Sedaie A, Pouladi N, Hosseinpourfeizi MA, et al (2014). Singlestrand conformational polymorphism analysis of a common single nucleotide variation in WRAP53 gene, rs2287499, and evaluating its association in relation to breast cancer risk and prognosis among Iranian-Azeri population. Med Oncol, 31, 168.

Somi MH, Mousavi SM, Rezaeifar P, Naghashi SH (2009) Cancer incidence among the Elderly population in the northwest of Iran: A population based study. Iranian $J$ Cancer Prev, 2, 117-126.

Taghavi A, Fazeli Z, Vahedi M, et al (2012). Increased trend of breast cancer mortality in Iran. Asian Pac J Cancer Prev, 13, 367-70.

Tripathi P, agrawal S (2006). Non-classical HLA-G antigen and its role in the cancer progression Cancer Invest, 24,178-86.

Tuncel T, Karagoz B, Haholu A, et al (2013). Immunoregulatory function of HLA-G in gastric cancer. Asian Pac J Cancer Prev, 14, 7681-4.

Veit TD, Chies JA (2009). Tolerance versus immune response - microRNAs as important elements in the regulation of the HLA-G gene expression. Transpl Immunol, 20, 229-31.

Wang X1, Jiang W, Zhang D (2013) Association of 14-bp insertion/deletion polymorphism of HLA-G gene with unexplained recurrent spontaneous abortion: a meta-analysis. Tissue Antigens, 81, 108-15.

Yz G, Q G, MH L, et al (2014). Association between human leukocyte antigen-G 14-bp insertion/deletion polymorphism and cancer risk: a meta-analysis and systematic review. Hum Immunol, 75, 827-32 